\title{
Das Tansanisch-deutsche Fachzentrum für Rechtswissenschaft (TGCL): Ausbildung künftiger Führungseliten für das östliche Afrika
}

\author{
Johannes Döveling*
}

\section{Einleitung}

Das Tansanisch-deutsche Fachzentrum für Rechtswissenschaft (Tanzanian-German Centre for Eastern African Legal Studies - TGCL) ${ }^{1}$ bildet in juristischen Master- und Promotionsprogrammen künftige Führungskräfte für das östliche Afrika aus. Das Fachzentrum wurde 2008 unter dem Namen „Tanzanian-German Centre for Postgraduate Studies in Law“ als Kooperationsprojekt der Universitäten Dar es Salaam in Tansania und Bayreuth gegründet. Der fachliche Schwerpunkt in Lehre und Forschung liegt auf dem Recht regionaler Integration unter besonderer Berücksichtigung des Rechts der im Jahr 2000 wiedergegründeten Ostafrikanischen Gemeinschaft (East African Community - EAC). Die Studierenden des TGCL stammen dementsprechend aus den fünf Partnerstaaten der EAC ${ }^{2}$ sowie aus Südsudan. ${ }^{3}$ Zusätzlich zum Studienprogramm trägt das Fachzentrum unter anderem durch regelmäßige internationale Fachtagungen zu aktuellen Rechtsfragen mit Relevanz für den ostafrikanischen Integrationsprozess zur Weiterentwicklung des EAC-Rechts bei.

\section{Institutioneller Rahmen}

\subsection{Förderprogramm}

Das Tansanisch-deutsche Fachzentrum wird im Programm „African Excellence - Fachzentren zur Eliteförderung" des Deutschen Akademischen Austauschdienstes (DAAD) gefördert. Die Mittel für dieses Programm werden vom Auswärtigen Amt als Maßnahme zur Stärkung der afrikabezogenen auswärtigen Kultur- und Bildungspolitik zur Verfügung gestellt.

Das Programm „African Excellence“ bezweckt die nachhaltige Schaffung von Ausbildungskapazitäten im Postgraduiertenbereich mit hoher regionaler und überregionaler Ausstrahlung an führenden afrikanischen Universitäten in Kooperation mit deutschen Universitäten. Damit soll die Ausbildung potentieller künftiger Führungseliten stärker als bisher di-

* Rechtsassessor Johannes Döveling, LL.M. (Cape Town), Wirtschaftsjurist (Univ. Bayreuth) ist Geschäftsführer des TGCL, Universität Bayreuth.

1 www.tgcl.uni-bayreuth.de.

2 Die Gründungsmitglieder der EAC sind Kenia, Tansania und Uganda. Burundi und Ruanda wurden im Jahr 2007 Mitglieder.

3 Ein Antrag Südsudans auf Mitgliedschaft ist bei der EAC anhängig. 
rekt in deren Heimatregionen erfolgen, um zum einen den regionalen Spezifika besser gerecht werden zu können und zum anderen die Abwanderung hochqualifizierter Fachkräfte in das Ausland („Brain Drain“) zu verhindern. Im Programm „African Excellence“ kombiniert der DAAD erstmals in größerem Rahmen die Projekt- mit der Individualförderung, denn den Fachzentren werden auch Mittel für von ihnen zu vergebende Stipendien zugewandt.

Die zunächst für fünf Jahre geförderten Fachzentren wurden im Jahr 2008 in einem Ausschreibungswettbewerb aus einer Vielzahl von Bewerbungen von einer unabhängigen Gutachterkommission ausgewählt. Nach der ersten Projektphase wurden alle Fachzentren in den Jahren 2011 und 2013, wiederum durch unabhängige Kommissionen, umfassend evaluiert. Der DAAD wird das TGCL aufgrund eines ausgesprochen positiven Begutachtungsergebnisses für weitere fünf Jahre fördern. Der Zielsetzung des Programmes entsprechend dient diese Förderphase auch der Etablierung eines Nachhaltigkeitskonzepts, um das Programm nach dem Auslaufen der Förderung fortsetzen zu können.

Innerhalb der Förderlinie „African Excellence“ bestehen derzeit sechs Fachzentren in Afrika, davon eines als Doppelzentrum:

\begin{tabular}{|l|l|l|}
\hline $\begin{array}{l}\text { Name des Fachzentrums und } \\
\text { Fachrichtung }\end{array}$ & Afrikanische Partnerhochschule & $\begin{array}{l}\text { Deutsche Partner- } \\
\text { hochschule }\end{array}$ \\
\hline $\begin{array}{l}\text { Kongolesisch-deutsches Fachzen- } \\
\text { trum für Mikrofinanz }\end{array}$ & $\begin{array}{l}\text { Université Protestante au Congo, } \\
\text { Kinshasa, Demokratische Republik } \\
\text { Kongo }\end{array}$ & $\begin{array}{l}\text { Frankfurt School of Fi- } \\
\text { nance and Manage- } \\
\text { ment }\end{array}$ \\
\hline $\begin{array}{l}\text { Ghanaisch-deutsches Fachzentrum } \\
\text { für Entwicklungsforschung }\end{array}$ & University of Ghana, Legon, Ghana & Universität Bonn \\
\hline $\begin{array}{l}\text { Namibisch-deutsches Fachzentrum } \\
\text { für Logistik }\end{array}$ & $\begin{array}{l}\text { Namibia Polytechnic of Science } \\
\text { and Technology, Windhuk, Nami- } \\
\text { bia }\end{array}$ & $\begin{array}{l}\text { Fachhochschule Flens- } \\
\text { burg }\end{array}$ \\
\hline $\begin{array}{l}\text { Südafrikanisch-deutsches Fachzen- } \\
\text { trum für Strafjustiz }\end{array}$ & $\begin{array}{l}\text { University of Western Cape, Kap- } \\
\text { stadt, Südafrika }\end{array}$ & $\begin{array}{l}\text { Humboldt-Universität } \\
\text { zu Berlin }\end{array}$ \\
\hline $\begin{array}{l}\text { Südafrikanisch-deutsches Fachzen- } \\
\text { trum für Entwicklungsforschung }\end{array}$ & $\begin{array}{l}\text { University of Western Cape, Kap- } \\
\text { stadt, Südafrika }\end{array}$ & $\begin{array}{l}\text { Ruhr-Universität Bo- } \\
\text { chum }\end{array}$ \\
\hline $\begin{array}{l}\text { Tansanisch-deutsches Fachzentrum } \\
\text { für Rechtswissenschaft }\end{array}$ & $\begin{array}{l}\text { University of Dar es Salaam, Dar } \\
\text { es Salaam, Tansania }\end{array}$ & Universität Bayreuth \\
\hline
\end{tabular}

Ein weiteres Fachzentrum im Fachbereich Bildungsforschung und -management wird derzeit an der Moi University, Eldoret, Kenia, in Kooperation mit der Universität Oldenburg und der Nelson Mandela Metropolitan University, Port Elizabeth, Südafrika, aufgebaut.

Regelmäßige Netzwerktreffen stellen den Austausch der Fachzentren untereinander sicher. Zudem besteht zwischen dem TGCL und dem Fachzentrum für Strafjustiz aufgrund ihrer fachlichen Nähe eine besonders intensive Beziehung. 


\subsection{Beteiligte Institutionen}

Das TGCL ist ein Kooperationsprojekt der Juristischen Fakultät der Universität Dar es Salaam mit der Universität Bayreuth, die mit ihrer Fakultät für Rechts- und Wirtschaftswissenschaften sowie mit ihrem Institut für Afrikastudien eingebunden ist.

Die 1975 gegründete Universität Bayreuth ist eine der führenden deutschen Universitäten in der Afrikaforschung. Sie zeichnet sich vor allem durch ihre breite multi- und interdisziplinäre wissenschaftliche Ausrichtung aus. Hierzu gehören auch rechtswissenschaftliche Forschung und Lehre, die inzwischen auf eine mehr als dreißigjährige Tradition zurückblicken. Der Bereich Recht in Afrika wird dabei durch eine außerplanmäßige Professur vertreten, zu deren Schwerpunktländern unter anderem Tansania und Kenia zählen.

Die University of Dar es Salaam School of Law ging aus der ältesten, 1961 gegründeten Juristischen Fakultät in Ostafrika hervor. Sie genießt hohe fachliche Reputation in der Region und gilt nach wie vor als eine der führenden Juristenausbildungsstätten Ostafrikas. Kenia, Sansibar, Tanganjika und Uganda kooperierten aufgrund der ihnen gemeinsamen früheren britischen Kolonialverwaltung nach der Unabhängigkeit Anfang der 1960er Jahre auch im Universitätswesen eng miteinander. Da ihre Rechtssysteme bis heute allesamt vom common law geprägt sind, liegt es nahe, dass dort schon seit damals Juristen aus allen diesen Staaten gemeinsam unterrichtet werden. Die University of Dar es Salaam School of Law drängte sich daher als Standort für das Ausbildungsprogramm des Fachzentrums geradezu auf. Dass bereits seit Beginn der 1980er Jahren ein reger wissenschaftlicher Austausch zwischen den beiden kooperierenden Fakultäten der Universitäten Bayreuth und Dar es Salaam stattfindet, kam dem Projekt insbesondere in der Aufbauphase zugute.

Seit Mitte 2014 ist an der Juristischen Fakultät der Universität Dar es Salaam zudem eine DAAD-Langzeitdozentur eingerichtet, die eng mit dem Fachzentrum verknüpft ist. Sie wird derzeit von $P D$ Dr. Tomasz Milej innegehalten. Der Inhaber der Langzeitdozentur ist Vollmitglied der Fakultät und ist dort eng in Forschung und Lehre eingebunden. Ein besonderes Augenmerk liegt dabei auf der wissenschaftlich reizvollen Verknüpfung europarechtlicher Fragen mit denjenigen zum Recht der EAC.

\subsection{Leitung}

Das TGCL wird durch das paritätisch besetzte Academic Committee geleitet, das alle grundsätzlichen und strategischen Entscheidungen fällt. Es setzt sich wie folgt zusammen:

\begin{tabular}{|l|l|}
\hline Name & Funktion \\
\hline Prof. Dr. Ulrike Wanitzek & TGCL-Projektleiterin, Universität Bayreuth \\
\hline Prof. Dr. Bonaventure Rutinwa & Dekan, University of Dar es Salaam School of Law ${ }^{4}$ \\
\hline
\end{tabular}

4 Vormalige Dekane seit Gründung des TGCL: Prof. Dr. Sifuni E. Mchome; Prof. Dr. Palamagamba J. Kabudi. 


\begin{tabular}{|l|l|}
\hline Name & Funktion \\
\hline Dr. Benedict T. Mapunda & TGCL-Koordinator, University of Dar es Salaam \\
\hline $\begin{array}{l}\text { Johannes Döveling, LL.M. (Cape } \\
\text { Town) }\end{array}$ & TGCL-Geschäftsführer, Universität Bayreuth ${ }^{6}$ \\
\hline
\end{tabular}

Als TGCL Programme Adviser übernimmt Prof. Dr. Dr. h.c. Gordon R. Woodman, University of Birmingham School of Law, eine Beratungsfunktion für das TGCL-Management.

Es existieren die folgenden weiteren Gremien zur Steuerung des TGCL: Einzelentscheidungen akademischer Natur werden vom Academic Governing Board getroffen, soweit nicht Organe der Universität Dar es Salaam zuständig sind. Neben Mitgliedern der dortigen juristischen Fakultät sollen dem Academic Governing Board auch hochrangige externe Juristen aus Justiz und öffentlicher Verwaltung angehören. Die Verantwortung für die akademischen Angelegenheiten wurde bewusst der tansanischen Partneruniversität übertragen, da die Studierenden des Fachzentrums LLM- und PhD-Abschlüsse der Universität Dar es Salaam erwerben und damit deren Regeln unterworfen sind. Zum anderen soll durch diese Aufgabenverteilung die institutionelle Nachhaltigkeit des Fachzentrums auch nach dem Auslaufen der Projektförderung gesichert werden.

Zusätzlich soll demnächst eine Academic Assembly mit beratender Funktion gegenüber dem TGCL-Management errichtet werden, in der die Dozenten und die Betreuer der Studierenden ihre Erfahrungen aus ihrer Tätigkeit für das TGCL einbringen.

\section{Studienprogramm}

\section{1. Überblick}

Das TGCL organisiert ein rechtswissenschaftliches Studienprogramm mit einem öffentlichrechtlichen Schwerpunkt auf dem Recht regionaler Integration unter besonderer Berücksichtigung des Rechts der East African Community. Die Studierenden des Fachzentrums sind dabei reguläre Studierende der Universität Dar es Salaam, kommen aber in den Genuss zahlreicher zusätzlicher intra- und interdisziplinärer Angebote. Diese umfassende Qualifizierung zielt darauf, neben vertieftem juristischem Fachwissen auch Kenntnisse zu vermitteln, die für eine spätere erfolgreiche Übernahme von Führungspositionen qualifizieren. Eigens für die Studierenden des TGCL wurde an der Universität Dar es Salaam der spezialisierte Abschluss LLM in Regional Integration and East African Community Law eingerichtet, der sich aus den Komponenten coursework and dissertation zusammensetzt. Absolventen des Doktorandenprogrammes erwerben einen allgemeinen $P h D$.

5 Vormaliger Koordinator: Dr. Kennedy Gastorn.

6 Vormalige Geschäftsführer: PD Dr. Harald Sippel; Florian Gonsior; Dr. Christian Kirchen. 


\subsection{Fachlicher Schwerpunkt}

In fachlicher Hinsicht konzentriert sich das TGCL auf das Recht der regionalen Integration. Als Referenzintegrationsbündnis wird dabei die East African Community (EAC) herangezogen, die derzeit - im Gegensatz etwa zur Southern African Development Community (SADC), der Tansania ebenso angehört - einen besonders dynamischen, zugleich aber mit signifikanten Implementierungsproblemen behafteten Integrationsprozess durchläuft.

Mit Unterzeichnung des Gründungsvertrages durch die Gründungspartner Kenia, Tansania und Uganda wurde die EAC zum 7. Juli 2000 ins Leben gerufen. Im Jahr 2007 wurden Burundi und Ruanda als weitere Partnerstaaten aufgenommen. Damit umfasst die EAC nunmehr neben den vom common law geprägten Gründungsstaaten auch civil law-Staaten, wobei in Ruanda derzeit ein Wandel hin zum common law stattfindet.

Die neu gegründete EAC kann an eine langjährige Tradition regionaler Kooperation in Ostafrika anknüpfen. Nach dem Ende der Kolonialzeit, die durch die enge Kooperation der von Großbritannien kontrollierten Gebiete geprägt war, wurde 1967 durch die unabhängig gewordenen Staaten Kenia, Tansania und Uganda die damalige East African Community als Wirtschaftsbündnis ins Leben gerufen. Kennzeichnend für diesen Zusammenschluss waren unter anderem gemeinsame Institutionen im Infrastruktur- und Staatsbankbereich. Als Hauptgründe für den Zusammenbruch dieser Gemeinschaft im Jahr 1977 werden unterschiedliche (wirtschafts-)ideologische Auffassungen der Mitgliedsstaaten, persönliche Rivalitäten zwischen den beteiligten Staatsoberhäuptern sowie ein Disput über die Verteilung der aus der Gemeinschaft erwachsenden Vorteile angeführt. Bereits in der 1984 geschlossenen Mediationsvereinbarung über die Aufteilung der Vermögenswerte der Gemeinschaft einigten sich die beteiligten Staaten darauf, künftig wieder enger miteinander zu kooperieren. Über die 1993 gegründete Permanent Tripartite Commission for East African Cooperation führte der Weg dann zur Wiedergründung der EAC durch den 1999 unterzeichneten Treaty for the Establishment of the East African Community. ${ }^{7}$

Dieser Gründungsvertrag sieht in Art. 5 (2) einen graduellen Integrationsprozess bis hin zur Etablierung einer politischen Föderation vor, der den klassischen Integrationsschritten regionaler Wirtschaftsbündnisse folgt: Zunächst wurde 2005 eine Zollgemeinschaft gegründet, 2009 dann mit dem Common Market Protocol zumindest der rechtliche Rahmen für einen gemeinsamen Markt geschaffen. ${ }^{8}$ Der äußerst ambitionierte Zeitplan sah zudem die Schaffung einer Währungsunion bis 2009 und als finales Ziel die Gründung der politischen Föderation in 2010 vor, deren Verwirklichung aus derzeitiger Sicht recht zweifelhaft erscheint.

7 Ausführlich zur Geschichte und Vorgeschichte der 1967 gegründeten East African Community: Sippel, Harald, Regional Integration in East Africa: A Legal Historical Overview, in: Gastorn, Kennedy, Harald Sippel and Ulrike Wanitzek (eds), Processes of Legal Integration in the East African Community, Dar es Salaam: Dar es Salaam University Press, 2011, 27-44.

8 Gastorn, Kennedy, The Legal Analysis of the Common Market of the East African Community as Market Freedoms in the Open Market Economy, Recht in Afrika 2011, 143-154. 


\subsection{Die Studierenden des $T G C L$}

Durch seine strenge fachliche Fokussierung auf das Recht der EAC ist das Studienprogramm des TGCL nicht nur für Juristinnen und Juristen aus Tansania, sondern auch für diejenigen aus den übrigen Partnerstaaten der Gemeinschaft äußerst attraktiv. Dementsprechend stammen die Studierenden aus insgesamt sechs Ländern: Neben den fünf Partnerstaaten der EAC ist seit kurzem auch Südsudan vertreten.

Das Fachzentrum legt großen Wert auf eine ausgewogene berufliche Mischung der Studierenden, von denen eine Vielzahl nach dem Erwerb ihres Bachelor-Abschlusses bereits berufstätig war. Auch eine ausgeglichene Geschlechterrepräsentanz wird angestrebt und weitgehend erreicht. Entscheidendes Auswahlkriterium bleibt jedoch eine durch einen herausragenden Abschluss des Bachelorstudiums und die mit der Bewerbung einzureichenden Research Proposals nachgewiesene exzellente Fachkompetenz. Mit nahezu allen Bewerberinnen und Bewerbern, die in einem Vorverfahren in die engere Wahl genommen wurden, wird ein persönliches Auswahlgespräch in ihrem Heimatland geführt. Neben der Verifikation der fachlichen Kompetenz dient dieses Gespräch vor allem der Begutachtung, ob ein Bewerber oder eine Bewerberin aufgrund des bisherigen gesellschaftlichen Engagements, des Charakters und der Bewerbungsmotivation zum Kreis derjenigen zählt, die potentiell künftig in Ostafrika verantwortlich Führungsaufgaben übernehmen können und wollen.

Es steht eine begrenzte Zahl von Stipendien für die LLM- und PhD-Studienprogramme zur Verfügung, die an besonders herausragende Kandidatinnen und Kandidaten vergeben werden. Die Stipendien decken neben den (relativ hohen) Studiengebühren auch die Lebenshaltungskosten sowie die Teilnahme an der Herbstuniversität in Europa und an der Studienfahrt innerhalb Ostafrikas. Seit dem Studienjahr 2014/15 nimmt das TGCL zudem Studierende in das Programm auf, die ihre Studiengebühren über ein externes Stipendium, über ihren Arbeitgeber oder über eigene Mittel finanzieren.

Sämtliche Absolventinnen und Absolventen haben unmittelbar nach Beendigung des Studienprogrammes eine Beschäftigung gefunden. Denjenigen, die bereits vor der Aufnahme ihres Studiums berufstätig waren, ermöglichte ihre Rückkehr mit einem zusätzlichen Abschluss regelmäßig einen Karrieresprung. Einige PhD-Absolventen des Fachzentrums unterrichten nach Abschluss ihres Studiums an Universitäten in Ostafrika und dienen so als Multiplikatoren ihres erworbenen Wissens. Andere berufliche Tätigkeitsfelder sind die Richter- und Anwaltschaft, der Auswärtige Dienst sowie oberste staatliche Behörden. Einige herausragende LLM-Absolventinnen und -Absolventen haben sich für eine Fortsetzung ihres Studiums im Rahmen eines PhD-Programmes entschlossen; mehrere von ihnen haben sich erneut für das TGCL entschieden und können so weiter im Bereich der von ihnen eingeschlagenen fachlichen Spezialisierung forschen. Ein prominentes Beispiel für die Übernahme von Führungsverantwortung durch TGCL-Alumni ist der derzeitige stellvertretende tansanische Außenminister, Hon. Mahadhi Juma Maalim, der kürzlich sein PhD-Studium erfolgreich abschloss. 


\subsection{LLM-Programm}

Im einjährigen LLM-Programm by coursework and dissertation wird den Studierenden systematisch ein solides Wissen im Recht der regionalen Integration vermittelt. Hierdurch werden sie in die Lage versetzt, Mechanismen regionaler Integration und deren rechtliche Umsetzung zu verstehen und kritisch zu hinterfragen.

Die drei jeweils 45stündigen University Courses sind das Herzstück der juristischen Wissensvermittlung des Studienprogrammes. Sie werden verblockt im ersten Semester des Studienjahres von mehreren Dozenten der Juristischen Fakultät der Universität Dar es Salaam gehalten, wobei der Inhaber der DAAD-Langzeitdozentur rechtsvergleichende Komponenten zum Europarecht und zum deutschen Recht einbringt. Neben den Kursen Law of Economic Integration und East African Community Law ist auch ein Kurs in Human Rights Law oder in Constitutional Law zu belegen. Die Einbeziehung des nationalen Verfassungsrechts ermöglicht es, auch Fragen des Verhältnisses von nationalem Recht zu supranationalem Recht gebührend zu behandeln. In allen Kursen finden Abschlussprüfungen statt, deren Bestehen Voraussetzung der Graduierung ist.

In ihrer LLM-Arbeit (dissertation) behandeln die Studierenden eine spezifische Frage des EAC-Rechts unter Berücksichtigung ihres nationalen Rechts. Während der gesamten Forschungs- und Schreibphase werden die Studierenden von einem Mitglied der Juristischen Fakultät der Universität Dar es Salaam betreut, das regelmäßig den Forschungsfortschritt mit seinen Studierenden bespricht.

\subsection{PhD-Programm}

Auch im PhD-Programm widmen sich die Studierenden im Rahmen ihrer Forschungsarbeit (thesis) einer Frage des EAC-Rechts, wobei nach Möglichkeit zwei nationalstaatliche Regelungssysteme zu betrachten sind. Das Programm ist als $P h D$ by research ausgestaltet, sodass keine coursework-Komponente vorgesehen ist. Dadurch können sich die PhD-Studierenden vollständig auf ihre Forschungsarbeit konzentrieren. Sie nehmen jedoch an den intra- und interdisziplinären Angeboten des TGCL teil, in denen sie auch die Möglichkeit haben, ihre methodischen Kenntnisse aufzufrischen und ihre Herangehensweise mit verschiedenen Dozenten kritisch zu diskutieren. Zusätzlich finden innerhalb der Fakultät regelmäßige Doktorandenseminare sowie monatliche Treffen der PhD-Studierenden mit dem TGCL Student Adviser statt. Sie dienen dem Austausch über den bisherigen Forschungsfortschritt, sodass sich die Studierenden bei etwaigen Herausforderungen nicht nur mit ihrem Betreuer, sondern auch mit Fachkollegen austauschen können.

Die Feldforschung der PhD-Studierenden wird durch eine großzügige Forschungspauschale als Bestandteil des Stipendiums gefördert. Im Einzelfall können darüber hinaus besonders leistungsstarken Studierenden auch Konferenzbesuche im außerafrikanischen Ausland ermöglicht werden. 
Die Doktorarbeiten werden in der vom TGCL herausgegebenen und bei Dar es Salaam University Press erscheinenden „TGCL Research Series“" veröffentlicht. ${ }^{9}$

\subsection{Schlüsselqualifikationen und interdisziplinäre Programmkomponenten}

Das TGCL verfolgt als erste Institution der Juristenausbildung für Postgraduierte in Ostafrika einen interdisziplinären Ansatz, um seinen Studierenden die für die Wahrnehmung von Führungsaufgaben benötigten Kenntnisse zu vermitteln. Hinzu kommt ein besonderes Betreuungsprogramm, durch das der Studienerfolg aller Studierenden sichergestellt werden soll.

Regelmäßig unterrichten Mitglieder der Rechts- und Wirtschaftswissenschaftlichen Fakultät der Universität Bayreuth in Dar es Salaam Betriebs- und Volkswirtschaftslehre. In den gewöhnlich viertägigen Kursen wird den Studierenden eine Einführung beispielsweise in Personalführung oder in Grundlagen der Betriebs- oder Volkswirtschaftslehre gegeben.

Zwei Seminare zur Forschungsmethodologie sorgen dafür, dass die Studierenden das nötige akademische Rüstzeug für ihre Forschung im Rahmen der Master- bzw. Doktorarbeit erwerben. In beiden Seminaren werden sie auch individuell gecoacht und haben damit Gelegenheit, sich von erfahrenen Wissenschaftlern frühzeitig über die Konzeption ihres Forschungsvorhabens beraten zu lassen.

Ein wesentlicher Faktor der Studienerfolge der TGCL-Studierenden ist deren umfassende und individuelle Betreuung in Studien- und Berufslaufbahnfragen durch den TGCL Student Adviser Dr. Steven Bwana, Richter am Court of Appeal of the United Republic of Tanzania. Er hat für die Studierenden aufgrund seines hohen Ansehens in ostafrikanischen Juristenkreisen und seiner vielfältigen Erfahrungen in Rechtspraxis und -theorie eine Vorbildfunktion. Richter Dr. Bwana wird bewusst nicht in die Lehr- und Verwaltungstätigkeit des Fachzentrums eingebunden, um den Studierenden als neutraler, externer Ratgeber zur Verfügung stehen zu können.

Zur Stärkung der Identifikation mit Deutschland und zur Vorbereitung auf den Deutschlandaufenthalt im Rahmen der Herbstuniversität nehmen die Studierenden zudem an einem Deutschkurs teil.

\subsection{Herbstuniversität und Studienreise}

Das Ausbildungsprogramm sieht eine TGCL-Herbstuniversität in Europa mit einem anspruchsvollen juristischen Fachprogramm mit Fokus auf dem Recht der Europäischen Union vor. Besucht werden die Universität Bayreuth, wo die Studierenden eine Einführung in das deutsche Recht erhalten, und Nürnberg mit dem Dokumentationszentrum Reichsparteitagsgelände sowie dem Memorium Nürnberger Prozesse. Weiterhin steht Berlin auf dem Programm, wo unter anderem der Deutsche Bundestag, das Auswärtige Amt (insbesondere

9 Die Forschungsthemen der PhD-Absolventen sind unten unter 4.1. dargestellt. 
die für die EU und für Afrika zuständigen Referate), das Justizministerium, die HumboldtUniversität sowie die tansanische Botschaft besucht werden. Im Jahr 2014 ist zudem erstmals ein Besuch in Brüssel vorgesehen, wo neben der Europäischen Kommission und dem Europäischen Parlament auch die Ständige Vertretung der Bundesrepublik Deutschland, die Vertretung des Freistaats Bayern und das Europabüro der Bayerischen Kommunen besucht werden. Hierdurch gewinnen die Studierenden wertvolle Einblicke in das Zusammenspiel der nationalen mit der europäischen Ebene.

Die durch die Herbstuniversität gewonnenen Erkenntnisse fügen sich dann wenige Monate später mit denjenigen der Studienexkursion in einen der Partnerstaaten der Ostafrikanischen Gemeinschaft zusammen, in deren Rahmen dortige juristische Institutionen besucht werden. Durch den frühen Zeitpunkt beider Exkursionen im Studienjahr können die Studierenden die Ergebnisse fruchtbringend für ihre Forschungsarbeiten verwerten.

\subsection{Alumni-Arbeit}

Das TGCL legt großen Wert darauf, langfristig den Kontakt zu seinen Graduierten zu halten und den Austausch der Alumni untereinander zu fördern. Hierfür finden regelmäßig Alumni-Treffen in allen Partnerstaaten der EAC statt, an denen nach Möglichkeit auch Mitglieder des TGCL-Managements teilnehmen.

Der Zusammenhalt der TGCL-Alumni untereinander wird dadurch bewiesen, dass sie aus eigener Initiative einen Alumni-Verein gründeten, der seitdem als Fundament der Alumniarbeit fungiert. Die im gesamten Ostafrika und darüber hinaus tätigen Alumni nutzen ihr Netzwerk auch im Alltag intensiv, beispielsweise bei der Bearbeitung grenzüberschreitender Fälle.

\subsection{Statistik}

Insgesamt wurden bisher 97 Studierende in das TGCL aufgenommen. Davon entfallen 23 auf das Promotionsprogramm (PhD) und 74 auf das Masterprogramm (LLM). Die Bewerberzahlen sind weitaus höher; im Schnitt kommen auf einen Studienplatz etwa acht Bewerbungen.

Die nachfolgend abgedruckte Tabelle gibt Aufschluss über die regionale Herkunft der Studierenden des TGCL.

\begin{tabular}{|l|c|c|c|c|c|c|c|}
\hline & Summe & Burundi & Kenia & Ruanda & Tansania & Uganda & Südsudan \\
\hline $\mathbf{2 0 0 8}$ & 10 & 0 & 0 & 0 & 10 & 0 & 0 \\
\hline $\mathbf{2 0 0 9}$ & 15 & 1 & 1 & 1 & 10 & 2 & 0 \\
\hline $\mathbf{2 0 1 0}$ & 15 & 1 & 4 & 1 & 7 & 2 & 0 \\
\hline $\mathbf{2 0 1 1}$ & 15 & 1 & 1 & 1 & 12 & 0 & 0 \\
\hline $\mathbf{2 0 1 2}$ & 14 & 1 & 3 & 1 & 6 & 3 & 0 \\
\hline $\mathbf{2 0 1 3}$ & 14 & 1 & 1 & 2 & 7 & 2 & 1 \\
\hline
\end{tabular}




\begin{tabular}{|l|c|c|c|c|c|c|c|}
\hline & Summe & Burundi & Kenia & Ruanda & Tansania & Uganda & Südsudan \\
\hline $\mathbf{2 0 1 4}$ & 14 & 1 & 4 & 1 & 5 & 1 & 2 \\
\hline Summe & $\mathbf{9 7}$ & $\mathbf{6}$ & $\mathbf{1 4}$ & $\mathbf{7}$ & $\mathbf{5 7}$ & $\mathbf{1 0}$ & $\mathbf{3}$ \\
\hline
\end{tabular}

\section{Forschung und Vernetzung mit der juristischen Praxis}

\subsection{Forschungsaktivitäten}

Die Studierenden des TGCL haben im Rahmen ihrer LLM- und PhD-Arbeiten bedeutende Forschungsergebnisse erzielt. Alle Arbeiten beruhen auch auf empirischen Forschungen.

Die am TGCL entstandenen Doktorarbeiten behandeln Themen höchster juristischer und gesellschaftlicher Relevanz:

So untersuchte beispielsweise Grace Kamugisha Kazoba aus Tansania den Rechtsrahmen für den Schutz von Verbrauchern vor den Auswirkungen von gefälschten und nicht normgerechten Arzneimitteln. ${ }^{10}$ Dabei bezog sie rechtsgebietsübergreifend das Recht des geistigen Eigentums, das Verbraucherrecht, internationales Handelsrecht sowie Menschenrechte mit ein. Mahadhi Juma Maalim aus Tansania analysiert in seiner Arbeit zu den Herausforderungen der regionalen Integration des innerhalb Tansanias teilautonomen Sansibars in die EAC eine politisch und juristisch hochaktuelle Fragestellung. ${ }^{11}$ Er kommt darin zu dem Ergebnis, dass der bisherige Rechtsrahmen eine Integration Sansibars in die EAC nicht ermöglicht und bietet zugleich konkrete Lösungsvorschläge an. Mit einer Untersuchung von Harmonisierungsmöglichkeiten der nationalen Einkommenssteuersysteme der EAC-Partnerstaaten hat Anatole Nahayo aus Burundi eine Grundlagenarbeit geschaffen, die erstmals einen systematischen Überblick über das Einkommenssteuerrecht in Ostafrika gibt. Seine Schlussfolgerung ist, dass die starken ökonomischen Unterschiede innerhalb der EAC eine ernstzunehmende Herausforderung für die Harmonisierung der Systeme darstellen. Im Ergebnis plädiert er für eine Vereinheitlichung der Systeme anstelle einer bloßen Harmonisierung, da nur so die erwünschten gesamtökonomischen Integrationsvorteile erreicht werden können. Lillian Mihayo Mongella aus Tansania untersuchte in ihrer Doktorarbeit das Recht auf Entschädigung für Opfer interner bewaffneter Konflikte in Ostafrika am Beispiel der Genozid-Opfer in Ruanda.

\subsection{Bibliothek}

Die gut sortierte TGCL-Bibliothek ermöglicht es den Studierenden, sich direkt am Fachzentrum eingehend mit der für ihre Master- oder Doktorarbeit relevanten Literatur zu befassen. Die Bibliothek umfasst etwa 1.200 Medien, die auch vom Personal der Fakultät und

10 Kazoba, Grace Kamugisha, Protection of Consumers and a Guard against Counterfeit and Substandard Pharmaceuticals in Tanzania, Dar es Salaam: Dar es Salaam University Press, 2013.

11 Maalim, Mahadi Juma, The United Republic of Tanzania in the East African Community. Legal Challenges in Integrating Zanzibar, Dar es Salaam: Dar es Salaam University Press, 2014. 
externen Studierenden rege genutzt werden. Sammlungsschwerpunkte sind das Recht der regionalen Integration, Verfassungsrecht, Menschenrechte und Rechtsvergleichung, ergänzt um Literatur zum Recht der Europäischen Union und zum deutschen Recht. Zudem sind die gängigen Gesetzes- und Entscheidungssammlungen und Sekundärliteratur aus den Mitgliedsstaaten der Ostafrikanischen Gemeinschaft vorhanden.

\subsection{Konferenzen}

Das TGCL veranstaltet seit seiner Gründung internationale juristische Konferenzen als einen wesentlichen Bestandteil seines Programms. Die jährlichen TGCL-Konferenzen bilden ein Kontinuum, zu dessen Ergänzung immer wieder weitere wissenschaftliche Konferenzen stattfinden, teils in Kooperation mit anderen Institutionen. Die Konferenzbeiträge werden in den Konferenzbänden der TGCL Series veröffentlicht. Themenschwerpunkte der letzten Jahre waren Menschenrechte, Verfassungsrecht unter besonderer Berücksichtigung der laufenden Verfassungsgebungsprozesse in Ostafrika sowie das Recht der regionalen Integration. Es ist dem TGCL ein besonderes Anliegen, mit diesen Konferenzen die Vernetzung von Wissenschaft und juristischer Praxis in Ostafrika zu stärken. Ein Teil der Redner sind daher hochrangige Persönlichkeiten aus denjenigen Institutionen, die für das behandelte Rechtsgebiet von besonderer Relevanz sind.

Einen wissenschaftlichen Höhepunkt bildete die Konferenz zum fünfjährigen Bestehen des TGCL. Sie fand im Oktober 2013 in Dar es Salaam zum Thema „Regional Integration and Law: East African and European Perspectives" statt. Während die ostafrikanische Perspektive von Rednern aus Tansania und Kenia dargestellt wurde, präsentierten Redner aus Frankreich und Deutschland ihre juristische Sicht auf den europäischen Einigungsprozess.

\subsection{Advanced Legal Training Seminars}

Ein wichtiges Element der Verknüpfung von Wissenschaft und Praxis am TGCL bilden die Advanced Legal Training Seminars. Dies sind verblockte Intensivseminare, in denen jeweils ein Rechtsgebiet, in dem grenzüberschreitende Sachverhalte eine bedeutende Rolle spielen, im Fokus steht. Dazu vermitteln je ein tansanischer und ein deutscher Jurist im Rahmen eines ,team-teaching'-Konzepts dieses Rechtgebiet innerhalb eines wissenschaftlichen Dialoges zum einen aus der Perspektive der Ostafrikanischen Gemeinschaft, zum anderen aus derjenigen der Europäischen Union. Solche Seminare fanden bereits zum Immaterialgüterrecht, zum EU/EAC-Recht sowie zum Wettbewerbsrecht statt. Die Advanced Legal Training Seminars finden bei Praktikern großen Anklang und gehören zusätzlich zum Pflichtprogramm für die Studierenden des TGCL. 


\section{Nachhaltigkeit}

Während der bis Ende 2018 laufenden Projektförderung durch den DAAD wird das Fachzentrum ein Nachhaltigkeitskonzept für die Zeit nach dem Ende der Förderung implementieren. Ziel ist es, die erfolgreiche Arbeit des TGCL langfristig fortsetzen zu können. Die erste Säule der Nachhaltigkeit besteht in der Verankerung des TGCL in der Fakultät durch die Schaffung dauerhafter institutioneller Strukturen ${ }^{12}$ unter gleichzeitiger schrittweiser Verlagerung von Kompetenzen von Deutschland nach Tansania. Ein Plan zur Sicherung der finanziellen Stabilität stellt die zweite Säule dar. Ziel ist es, die Kernkomponenten des Programmes auch nach dem Auslaufen der zehnjährigen Anschubfinanzierung durch den DAAD sicherzustellen. Die wesentlichen Erlösquellen werden dabei die Einnahmen aus Studiengebühren sowie aus Advanced Legal Training Seminars sein.

12 Siehe hierzu auch die Darstellung der Organe des TGCL, oben unter 2.3. 